\title{
Quantitative Trait Loci Underlying Partial Resistance to Cerco- spora sojina Race 2 Detected in Soybean Seedlings in Green- house Assays
}

\author{
Hemlata Sharma ${ }^{1,2}$ and David A Lightfoot ${ }^{2,3^{*}}$ \\ ${ }^{1}$ Dept of Plant Breeding \& Genetics, Rajasthan College of Agriculture, MPUAT, Udaipur, India; ${ }^{2}$ Department \\ of Plant, Soil and Agricultural Systems, Southern Illinois University, Carbondale, IL, 62901, USA; ${ }^{3}$ The Illinois \\ Soybean Center, Southern Illinois University, Carbondale, IL, 62901, USA
}

Received: June 30, 2013 / Accepted: March 18, 2014

\begin{abstract}
Cercospora sojina (Hara), an air-borne pathogen, infects soybean [Glycine max (L.) Merr.] leaves causing frog-eye leaf spot (FLS). Three major genes (Rcs I-3) underlie resistance to the major races of FLS but two were not yet mapped. In addition quantitative trait loci provide partial resistance to many strains. FLS race 2 was an isolate first collected in the 1950's when damaging FLS first arose. 'Essex' was partially resistant while 'Forrest' was partially susceptible to mixed races of FLS. The objective here was to identify quantitative trait loci underlying resistance to FLS race 2 in the greenhouse using recombinant inbred lines (RILs) derived from the cross of Essex by Forrest. C. sojina race 2 (ATCC 44531) was used to induce leaf symptoms on one hundred $F_{5: 14}$ RILs derived from the cross of Essex by Forrest. The leaf symptoms were measured at 21 days after manual infestation by wounding (dai) and again at $\mathbf{4 2}$ dai to show resistance to reinfestation of new leaves from the primary lesions without wounding. Bags over leaves were not used to better simulate field conditions. However, there was no significant correlation between FLS severity at 21 and 42 dai $(r=0.08$ and $P=0.005)$. At 21 dai there was a strongly significant QTL near Satt319 on LG C2 (chromosome 7; LOD 3.8; $\mathbf{R}^{2}$ 52\%) where the Essex allele reduced leaf symptoms by 0.7 units. At 42 dai there was a strongly significant QTL near Satt632 on LG A2 (chromosome 8 ; $\mathbf{R}^{2}$ was $15 \%$; LOD was 3.6 ) where the Essex allele reduced leaf symptoms by 0.4 units. Neither locus mapped
\end{abstract}

*Corresponding author: ga4082@siu.edu to the location of Rcs3. By ANOVA thirteen additional minor loci were detected on LGs A1, B1, F, G, H, I, J, K, L, M and $O$. At two loci (LG $B 1$ and O) the Forrest allele appeared to reduce FLS at both 21 and $\mathbf{4 2}$ dai. Eight loci may have reduced FLS at 21 dai $\left(0.006<\mathrm{P}<0.049 ; 4 \%<\mathrm{R}^{2}>9 \%\right)$ of which 5 had beneficial alleles from Forrest. Seven loci may have reduced FLS at 42 dai $\left(0.001<P<0.04 ; 4 \%<R^{2}<15 \%\right)$ of which 4 had beneficial alleles from Forrest. Therefore, quantitative resistance to race 2 of FLS was inferred to have major loci contributions from Essex and minor loci contributions from both Forrest and Essex. Resistance was dependent on plant age. Breeding and selection for FLS will be complex and may be more efficient with the markers, germplasm and models of inheritance reported here.

Keywords: Cercospora; tolerance; QTL; resistance; soybean; frog-eye leaf spot.

\section{Introduction}

Frogeye leaf spot (FLS), caused by the fungus Cercospora sojina (Hara), has been an important soybean disease, that has caused both seed yield losses and seed quality deterioration (Wrather et al., 1996; 2003). Yield reductions in the range of 10 to $60 \%$ due to FLS have been reported. The use of resistant cultivars was the most efficient and cost effective means of controlling this disease (Mian et al., 2009). However, the rise
ATLOS Publishing, LP
This is an Open Access article distributed under the terms of the Creative Commons Attribution License (http://creativecommons.org/licenses/by/3.0/), which permits unrestricted use, distribution, and reproduction in any medium, provided the original work is properly cited. 
of dozens of new C. sojina races (Yorinori, 1992; Mian et al., 2009) has reduced the effectiveness of the three major genes deployed to date. A new approach to the search for sources of resistance may involve loci underlying partial resistance and their incorporation into breeding programs (Gravina et al., 2004; Mian et al., 2008; 2009).

Three single genes conditioning resistance to C. sojina were; Rcs 1 from 'Lincoln' that conferred resistance to race 1 (Athow and Probst, 1952); Rcs2 for resistance to race 2 identified from cultivar 'Kent' (Athow et al., 1962); and Rcs3 from 'Davis' that was found to condition resistance to race 5, among others (Boerma and Phillips, 1983; Phillips and Boerma, 1982; Yorinori, 1992). Dominant genes for resistance to race 5 that were not alleles of Rcs $7-3$ were found in 'Peking', 'Ransom', 'Stonewall', and 'Lee' (Pace et al., 1993; Baker et al., 1999).

On its release (Smith and Camper, 1973) 'Essex' was reported to show partial resistance to both frogeye leaf spot (caused by $C$. sojina $K_{\text {.) }}$, and purple seed stain disease [caused by $C$. kikuchii (Mastsumoto \& Tomoyasu) M.W. Gardner]. Essex had, among its immediate parents, Lee, a potential source of Rcs 3 like loci. 'Forrest' had, among its immediate ancestors, Peking the source of an Rcs 3 like locus. Forrest also had, on either side of its phylogeny, Lee and its sibling line 'D49-2491', both potential sources of Rcs3 like loci (Hartwig and Epps, 1973; Lightfoot et al., 2005). Both Essex and Forrest shared cultivars 'CNS' and 'S100' as ancestors, potential sources of FLS resistance genes. Essex was reported resistant to FLS race 2 in the field but susceptible to FLS race 5 (Phillips and Boerma, 1981). Further, race 2 of C. sojina appeared (Athow et al., 1962) in the late-1950s, in the Carolinas and Mid-West of the USA. Therefore, it is likely Essex, Forrest and their parents were unintentionally selected for quantitative resistance to FLS race 2 in the field. However, whilst it was reported that Essex had field resistance to FLS it was not reported whether Forrest was resistant (Hartwig and Epps, 1973) and it was later shown not to carry Rcs3 (Missaoui et al., 2007).

Therefore, the objective of this study was to identify major loci underlying resistance and/or polygenes underlying QTL for partial resistance or tolerance to the foliar symptoms of FLS race 2 under controlled conditions in the greenhouse using seedlings of an advanced RIL population.

\section{Materials and Methods}

\section{Materials}

The experimental material in the publicly released population EF94 included ninety four $F_{5: 14}$ RILs (Hnetkovsky et al.,1996; Lightfoot et al., 2005) derived from the cross of Essex (Smith and Camper, 1973) x Forrest (Hartwig and Epps, 1973) and the two parents. In addition the 6 RILs removed from EF94, RILs 95-100 were returned to the population tested. The susceptible check used was 'Blackhawk' and the resistant check used was 'Kent'. The strain of race 2 was provided by Dr. J. Phillips through the American type culture collection (ATCC) as strain number 44531. It was last deposited in 2008 . The strain was received desiccated and was grown on V8 media plates (Kent et al., 2008) at
$22{ }^{\circ} \mathrm{C}$ in the dark. SIUC retains a duplicate, frozen, desiccated isolate for distribution on request. Alternately the strain can be obtained from the ATCC.

\section{Infestations with C. sojina}

Plates used for inocula were washed with distilled water and $10 \mu \mathrm{l}$ of this was used for spore count on a hemo-cytometer under a microscope. Spore counts of $10^{4}$ spores per $\mathrm{cm}^{3}$ were used. The one hundred RILs were planted in $15 \mathrm{~cm}$ square pots the greenhouse at the Southern Illinois University Horticulture Research Center in Carbondale, IL. Parents and non-inoculated control plants were included in the experiments. All plants were sown in sterilized 1:1 (v/v) of sand and soil and grown to the V3 stage (about 21 days; Fehr and Caviness, 1977). The first fully expanded leaves of 3 plants per genotype were selected to be infested by $\mathrm{C}$. sojina. Three more plants per genotype were selected for non-inoculated controls (natural infestation). Across both repeats of the experiment 6 inoculated plants and 6 noninoculated plants were scored. Plants were arrayed in randomized complete blocks. The plants to be infested were inoculated by rubbing the youngest full expanded leaflets with 600 mesh CarborundumTM paper. Leaflets were sprayed to runoff with the spore solution. Infested leaves were not bagged as in Mian et al (2008) so symptoms would develop more slowly and reflect field infestations to a greater degree. Experiments were conducted from October to December in 2008 and repeated in full from October to December in 2011 . Plants were grown with a $14 \mathrm{~h}$ photoperiod and $10 \mathrm{~h}$ of darkness using sunlight supplemented by growlights providing 1,500-2,000 mol photons $\mathrm{m}^{-2}$ $\mathrm{s}^{-1}$. Greenhouse air temperature ranged from $20 \pm 2^{\circ} \mathrm{C}$ at night to $27 \pm 2{ }^{\circ} \mathrm{C}$ during the day. Humidity ranged from $75-85 \%(v / v)$ as judged by indicator cards. In total 6 plants per genotype contributed to the trait means. The mean disease severity (DS) of the non-inoculated plants was subtracted from the infested plant mean for each genotype.

FLS DS was rated at 21 days after inoculation (dai; 42 days after germination), and 42 dai. The DS rating was determined on the basis of the degree of leaf damage of the whole canopy (chlorosis/necrosis) on each plant, and was rated on a scale of 0 to 9 ( 0 was $0 \% / 0 \% ; 1$ was $1-10 \% / 1-5 \% ; 2$ was $10-20 \% / 6-$ $10 \%$; 3 was $20-40 \% / 10-20 \%$; 4 was $40-60 \% / 20-40 \%$; 5 was $>60 \% />40 \%$ of leaf surface chlorosis/necrosis, respectively; 6 was up to $33 \%$ premature defoliation; 7 was up to $66 \%$ premature defoliation; 8 was more than $66 \%$ premature defoliation; and 9 was premature plant death). Means, plus and minus the standard error of the means (SEMs), were reported. The DS scores for cultivar Blackhawk (susceptible check) was 5 at 21 dai and 5 at 42 dai. The DS scores for Kent (resistant check) was 0 at 21 dai and 0 at 42 dai.

\section{Trait Analyses}

Since the assay was conducted under controlled conditions in the greenhouse the broad sense heritability (h2) of DS was calculated from variance components as:

$$
\mathrm{h} 2=\sigma 2 \mathrm{~g} /(\sigma 2 \mathrm{~g}+\sigma 2 \mathrm{e})
$$


Where $\sigma 2 \mathrm{~g}=$ genotypic variance and $\sigma 2 \mathrm{e}=$ error variance. Pearson's correlation coefficient analysis was performed on the mean trait data in order to test for correlations among traits. RIL's and parent trait mean comparisons were made by LSD.

\section{Mapping Quantitative Trait Loci}

A linkage map was created using MAPMAKER-EXP 3.0 (Lander et al., 1987); the (ri-self) genetic model; cM in Haldane units and a two point analysis. The RIL population was used to map a total of 413 markers, including 238 satellite markers and 177 other DNA markers (Kassem et al., 2006; Yesudas et al., 2010). The $\log 10$ of the odds ratio (LOD) for grouping markers was set at 3.0 and the maximum distance was $50 \mathrm{cM}$. Conflicts were resolved in favor of the highest LOD score after checking the raw data for errors. Marker orders within groups were determined by putting them in increasing order of sequence number in the soybean genome (Schmutz et al., 2010).

The map and disease scores were analyzed with ANOVA (SAS, Cary, NC) by single point analyses. Markers were listed if they were significant at $0.001<\mathrm{P}<0.05$ at either 21 or 42 dai. Markers that were unlinked, or in marker sparse areas of the map, were considered to be independent tests (Kassem et al., 2006; Shultz et al., 2007; Yesudas et al., 2010). Composite interval analysis used WinQTL Cartographer (version 2.0; Basten et al., 2001) for QTL mapping and estimation of locus effects as described previously (Kassem et al., 2006; Shultz et al., 2007; Yesudas et al., 2010).

\section{Results}

\section{Trait Heritability}

The broad sense heritability for FLS DS in the RILs was 83.9 $\%$ at 21 dai and $64.8 \%$ at 42 dai. The genotype $x$ environment (GxE) interaction was not significant since the greenhouse conditions were closely controlled. That was used as the justification to use the mean data (Niiti et al. 2001). The correlation between 21 dai and 42 dai mean DS across the population was $r=0.08$ suggesting the traits were largely independent.

\section{Trait Distributions}

DS from the replication and repeats at either 21 dai or 42 dai showed similar severity and were highly correlated so data were pooled and those means used for further analyses (Fig. 1). At 21 dai the distribution of mean DS was nearly normal and neither significantly skewed or kurtotic (Fig. 1). The mean DS distribution ranged from 0 to 5. The mean DS+SEM for Essex was $1.85+0.02$ and significantly different $(P<0.001)$ from Forrest was $3.8+0.02$ as judged by LSD. The 20 most resistant and 13 most susceptible lines were segregation events that were significantly $(P<0.05)$ better than Essex or worse than Forrest (hereafter transgressive segregants). At 42 dai the distribution of mean DS was positively skewed $(P<0.05)$ towards resistance. The distribution was continuous and had a significant negative kurtosis that reflected a flattened distribution (Fig. 1). The
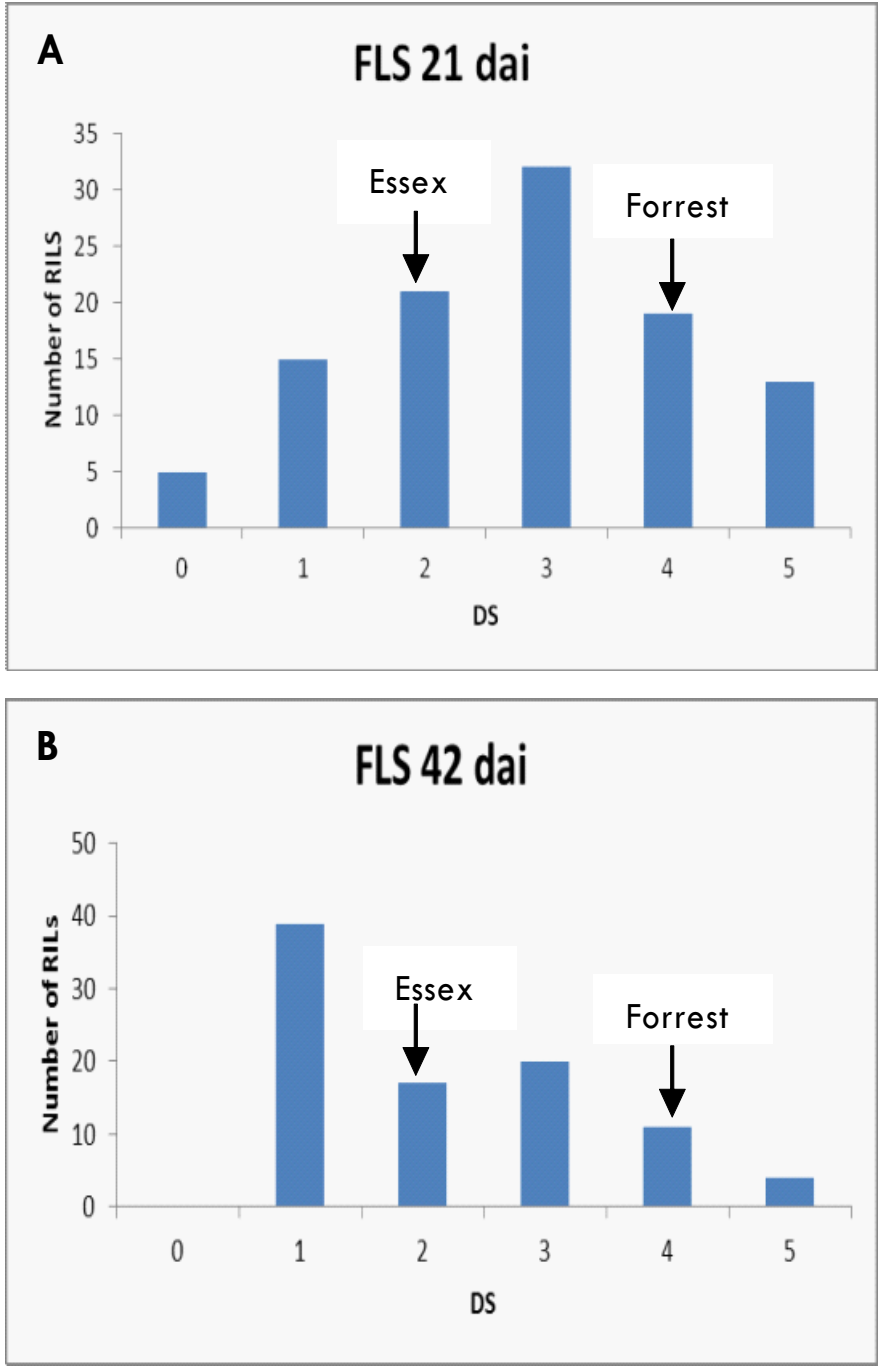

Figure 1. Frequency distributions of mean DS among RILs from the ExF cross in greenhouse seedling assays at 21 dai (A) and 42 dai (B). The ranges into which Essex $(E)$ and Forrest $(F)$ mean scores fell are arrowed.

mean DS distribution ranged from 0 to 5 , similar to that seen for bigenic resistance to SCN (Lightfoot, 2008). The mean DS+SEM for Essex was $1.65+0.02$ and significantly different $(P<0.001)$ from Forrest that was $2.45+0.02$. The 19 most resistant and 3 most susceptible lines were significant $(P<0.05)$ transgressive segregants.

\section{Major QTL Defection}

At 21 dai there was a major QTL on near Satt319 on LG C2 (chromosome 7; LOD was 3.8; R2 was 52\%) where the Essex allele reduced FLS leaf symptoms by 1.3 DS units (Table 1; Fig. 2). At 42 dai there was a major QTL near Satt632 on LG A2 (chromosome 8; R2 was 15\%; LOD was 3.6) where the Essex allele reduced FLS leaf symptoms by 0.46 DS units. The two loci represented major gene effects and underlay a large portion of the variation in trait distributions (Fig. 1). Notably, both loci derived beneficial alleles from Essex, the most resistant parent. 


\section{Minor QTL Detection}

In addition to the two major loci there were thirteen minor loci that may be associated with resistance to FLS race 2 detected by ANOVA (Table 2) at $0.001<P<0.05$. Some of these loci had beneficial alleles from Forrest. At two loci the Forrest allele appeared to reduce FLS at both 21 and 42 dai. The first locus was associated with Satt444 on LG B 1 (chromosome 11) at 85.9 $\mathrm{CM}$. The locus explained $6 \%$ of the variation in FLS at 21 dai $(P=0.017)$ with the Forrest allele reducing $D S$ by up to 0.59 units. A locus in the same region explained $12 \%$ of the variation in FLS at 42 dai $(P=0.008)$ with the Forrest allele reducing DS by up to 0.66 units. The second locus was only weakly associated with variation in FLS near Satt446 on LG L (chromosome 19; $\mathrm{P}<0.049$ ).

Six loci were solely associated with reduced FLS at 21 dai $\left(0.006<P>0.045 ; 4 \%<R^{2}>9 \%\right)$ of which 5 had beneficial alleles from Forrest. The first locus was associated with SCAR CFR2 on LG F (chromosome 13) at $1.1 \mathrm{cM}$. The locus explained $9 \%$ of the variation in FLS at 21 dai $(P=0.006)$ with the Forrest allele reducing DS by up to 0.75 units. The second locus was associated with SCAR CGG 116 on LG G (chromosome 18) at 10.1 $\mathrm{CM}$. The locus explained $6 \%$ of the variation in FLS at 21 dai $(P=0.006)$ with the Essex allele reducing $D S$ by up to 0.66 units. The third, fifth and sixth loci listed in Table 2 were only weakly associated with variation in FLS The fourth locus was associated with Satt249 on LG J (chromosome 16) at $12.3 \mathrm{cM}$. The locus explained $8 \%$ of the variation in FLS at 21 dai $(P=0.009)$ with the Essex allele reducing DS by up to 0.77 units.

Five loci were only associated with reduced FLS at 42 dai $\left(0.001<P>0.042 ; 4 \%<R^{2}>15 \%\right)$ of which 4 had beneficial alleles from Forrest. The first locus was associated with Satt276 on LG Al (chromosome 5) at $17.2 \mathrm{cM}$. The locus explained $13 \%$ of the variation in FLS at 42 dai $(P=0.007)$ with the Forrest allele reducing DS by up to 0.64 units. The second locus was associated with Satt589 on LG A2 (chromosome 8) at $34.0 \mathrm{cM}$. The locus explained $11 \%$ of the variation in FLS at 42 dai $(P=0.01)$ with the Forrest allele reducing $D S$ by up to 0.64 units. The third locus was associated with Satt440 on LG I (chromosome 20) at $112.7 \mathrm{cM}$. The locus explained 15\% of the variation in FLS at 42 dai $(P=0.001)$ with the Essex allele reducing DS by up to 0.95 units. The fourth locus was associated with both Satt555 and Sat_116 on LG K (chromosome 9) at 42.7 and $52.3 \mathrm{cM}$ respectively. The locus explained $11 \%$ of the variation in FLS at 42 dai $(P=0.009)$ with the Forrest allele reducing DS by up to 0.67 units. The fifth locus was minor in effect $(P=$ 0.045 ) and was associated with Satt259 on LG O (chromosome 10) at $39.8 \mathrm{cM}$.

\section{Discussion}

The broad sense heritability for FLS DS was high at 21 dai and moderate at 42 dai. The earlier date may be higher in heritability because the trait was the direct effect of inoculation. In comparison the later FLS DS score was the product of a more natural inoculation and indirect infestation of new leaves from diseased leaves. The correlation between 21 dai and 42 dai mean DS suggested the traits were largely independent and that the later DS score was the product of different resistance response mechanisms. Infection after wounding measured at 21 dai eliminates or reduces the effectiveness of resistances derived from barriers like the cuticle, anti fungal waxes, and even cell wall integrity.

Trait distributions differed in normality, skewness and kurtosis, again suggesting the traits were underlain by different response mechanisms. The most resistant transgressive segregants for both DS scores were ranked in order EF 7, 28, 2, 8, 61, 86. These lines would be useful parents for crosses designed to select loci underlying quantitative resistance to FLS (Gravina et al., 2004; Mian et al., 2009).

Quantitative resistance to C. sojina race 2 was shown to be underlain by two major QTL in the seedlings of EF94. The two loci were effective at different stages of seedling development, suggesting they were conditional QTL. The locations of the QTL suggested the loci are not allelic to Rcs3 (LG J). The locations of Rcs 1 and Rcs 2 had not been determined by 2011, so the loci detected might be allelic to these qualitative resistance loci. However, in view of the Lee ancestry of Essex, the loci are not allelic to Rcs 1-3 (Pace et al., 1993; Baker et al., 1999). Therefore, the loci represent new quantitative resistance loci possibly underlying a more broad and durable type of resistance found with other diseases (Hnetkovsky et al., 1996). The loci mapped to locations containing several other resistance loci for SCN, SDS, insect herbivory and seed yield (Yuan et al., 2002; Kassem et al., 2006; Yesudas et al., 2010). Since Essex provided the beneficial allele at the QTL on LG C2 the resistance alleles to FLS, SDS and seed yield were in coupling. However, on LG A2 resistance alleles to FLS and insect herbivory were linked in repulsion to $\mathrm{SCN}$ resistance. Therefore, the major loci detected here may be useful for the genetic improvement of resistance to $C$. sojina by selection of new recombination events to break repulsion and create QTL stacks to increase resistance to C. soiina.

The minor loci detected here were on eleven linkage groups (A I, B 1, F, G, H, I, J, K, L, M and O). Minor loci might represent type II errors. Alternately the loci might be in sparse areas of the map. A third possibility is the loci were major genes but with alleles that were not very diverged in Essex and Forrest. A fourth

Table 1. Detection of QTL underlying resistance to Cercospora sojina at 21 dai and at 42 dai by CIM.

\begin{tabular}{llllllllll}
\hline Trait & QTL & L.G. & Marker/Interval & $\begin{array}{l}\text { Peak cM } \\
\text { position }\end{array}$ & LOD & Additive & $\mathbf{R}^{2}$ & $\begin{array}{l}\text { Essex allelic } \\
\text { mean } \pm \text { SEM (n) }\end{array}$ & $\begin{array}{l}\text { Forrest allelic } \\
\text { mean } \pm \text { SEM (n) }\end{array}$ \\
\hline FLS 21 dai & 1 & C2 & Satt319-Satt079 & 120 & 3.8 & -0.58 & 0.52 & $2.44 \pm 0.01(44)$ & $3.80 \pm 0.01(45)$ \\
FLS 42 dai & 2 & A2 & Satt632-A2D8 & 50 & 3.6 & -0.92 & 0.15 & $1.93 \pm 0.01(47)$ & $2.36 \pm 0.01(38)$ \\
\hline
\end{tabular}


start

Satt520

Satt291

Satt305

Satt376

Satt363

Satt286

Satt277

Satt134

Satt319

Satt489

Satt079

Satt307

Satt202

Satt316

Satt371

Satt357

Satt371

Satt357

end o

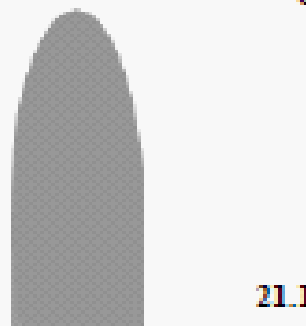

42.4

H100B $10 \mathrm{~b}$

H100B 10a

OVI 15_400

69.665

OH023_70O

97.8

98.1

101.8

107.59

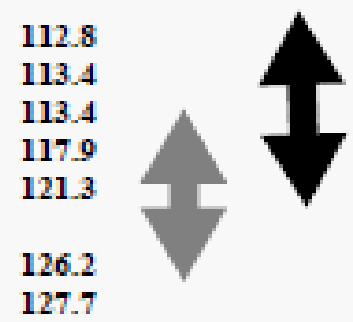

1455

151.9

ATACT 310

145.5

151.9

157.9
A2DS

BLT65

Sat 162

Sitt632

Sott 424

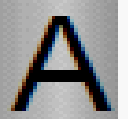

60.6

67.6

Sat 129

Sittos?

84. 1

876

A117_1

1043

Sat 040

Sat_097

Satt133

118.6

122.1

125.4

Satt409

1456
153.5

1456
153.5

72

24.1

73

\section{9}

478

481

493

519

1.5

5.4

\author{
125.4
}

B31L10

End

1655

1657

Figure 2. Locations of the major QTL found in the Essex by Forrest population on linkage groups A2 (42 dai) and C2 (42 dai) for FLS DS (black arrows). Also shown are QTL for HG Type 0 (black stippled arrow); seed yield (light grey solid arrow); and herbivory by Japanese beetle (dark grey solid arrow). The size of the arrow reflects the interval significantly associated by QTL Cartographer or Mapmaker at LOD > 2.0 or ANOVA at $\mathrm{P}<0.001$. 
possibility is that loci for resistance to other FLS races contribute some quantitative resistance to race 2 .

The minor loci might be candidates for the non-allelic dominant genes for resistance to FLS race 5 or 6 that were found in Peking, CNS, S100, D49-2491 and Lee (Pace et al., 1993; Baker et al., 1999).

\section{Conclusions}

Resistance to race 2 of FLS was inferred to be a quantitative trait with major loci contributions from Essex and minor loci contributions from both Forrest and Essex. The genes were not in the regions of $R \operatorname{cs} 3$ but one or two of them might be allelic to $R \operatorname{cs} 1$ or Rcs2. However, it is more likely is that race 2 resistance preceded the development of Rcs genes and was quantitative (Smith and Camper, 1973; Phillips and Boerma, 1981). Since all major loci and most minor loci were active at only one stage of seedling development it was inferred that this quantitative resistance to FLS was conditional upon wounding state or growth stage. The method recommended by Mian et al., (2008) for qualitative resistance was quite different since it does not wound the leaf, wraps the sprayed leaf in a plastic bag for $48 \mathrm{~h}$ and scores FLS only at 14 dai. Therefore, the three different methods may not detect the same loci. However, the breeding and selection for FLS may be more efficient with the markers, transgressive segregants and models of inheritance reported here.

Alleles from Essex are common among modern US cultivars (Lam et al., 2010). Alleles from Forrest can be selected in derived lines like Hartwig and it's derivatives (Anand, 1992). Recombination events breaking the repulsion between resistance to FLS and resistance to SCN would be useful advances for breeding. The resources available for genomics from Forrest may assist in the isolation of genes underlying the major quantitative trait loci for resistance to many FLS races (Lightfoot, 2008).

\section{Acknowledgements}

The authors thank Dr. P. Gibson, O Myers Jr. and M. Schmidt for assistance with germplasm development and maintenance from 1991-2000. We thank the BOYSCAST program and the government of India for the fellowship to HS.

\section{References}

Athow KL and AH Probst (1952) The inheritance of resistance to frogeye leaf spot of soybeans. Phytopathology 42: 660-662.

Athow KL, AH Probst, CP Kartzman, and FA Laviolette (1962) A newly identified physiological race of Cercospora sojina on soybean. Phytopathology 52: 712-714.

Anand SC (1992) Registration of 'Hartwig' soybean. Crop Sci 32: 1060-1070.

Baker WA, DB Weaver, J Qu, and PF Pace (1999) Genetic analysis of frogeye leaf spot resistance in PI54610 and Peking soybean. Crop Sci 39: 1021-1025.

Basten CJ, BS Weir, and Z Zeng (2001) QTL Cartographer Version 2.0. Raleigh, NC: Department of Statistics, North Carolina State University, USA.

Boerma HR and DV Phillips (1983) Genetic implications of the suscep- tibility of Kent soybean to Cercospora sojina. Phytopathology 74: 1666-1668.

Fehr WR and CE Caviness (1977) Stages of soybean development. Special Report 80. Ames, lowa: Cooperative Extension Service, Agriculture and Home Economics Exp Stn lowa State University 11: 929-931.

Gravina G, C Filho, S Martins, M Moreira, E de Barros, and C Cruz (2004) Multivariate analysis of combining ability for soybean resistance to Cercospora sojina Hara. Genet. Mol. Biol. 27: 395-399.

Hartwig EE and JM Epps (1973) Registration of Forrest soybeans. Crop Sci 13: 287.

Hnetkovsky N, SJC Chang, TW Doubler, PT Gibson, and DA Lightfoot (1996) Genetic mapping of loci underlying field resistance to soybean sudden death syndrome (SDS). Crop Sci 36: 393-400.

Kassem MA, J Shultz, K Meksem, Y Cho, AJ Wood, MJ lqbal, and DA Lightfoot (2006) An updated 'Essex' by 'Forrest' linkage map and first composite interval map of QTL underlying six soybean traits. Theor Appl Genet 1 13: 1015-1026.

Kent CR, P Ortiz-Bermúdez, SS Giles, and CM Hull (2008) Formulation of a defined $V 8$ medium for induction of sexual development of Cryptococcus neoformans. Appl Environ Microbiol. 74: 6248-6253.

Lam HM, X Xu, X Liu, WB Chen, GH Yang, et al., (2010) Resequencing of 31 wild and cultivated soybean genomes identifies patterns of genetic diversity and selection. Nat Genet 42: 1053-1059.

Lander E, P Green, J Abrahamson, A Barlow, M Daley, S Lincoln, and L Newburg (1987) MAPMAKER: An interactive computer package for constructing primary genetic linkage maps of experimental and natural populations. Genomics 1: 174-181.

Lightfoot DA, VN Nijiti, PT Gibson, MA Kassem, MJ lqbal, and K Meksem (2005) Registration of the Essex X Forrest recombinant inbred line mapping population. Crop Sci 45: 1678-1681.

Lightfoot DA (2008) Soybean genomics: Developments through the use of cultivar Forrest. International Journal of Plant Genomics 2008:1 22. doi: $10.1155 / 2008 / 793158$.

Mian MAR, AM Missaoui, DR Walker, DV Phillips, and HR Boerma (2008) Frogeye leaf spot of soybean: A review and proposed race designations for isolates of Cercospora sojina Hara. Crop Sci 48: 14-24.

Mian R, J Bond, T Joobeur, A Mengistu, W Wiebold, G Shannon, and A Wrather (2009) Identification of soybean genotypes resistant to Cercospora sojina by field screening and molecular markers. Plant Dis 93: 408-411.

Missaoui AM, DV Phillips, and HR Boerma (2007) DNA marker analysis of 'Davis' soybean and it's descendants for the Rcs3 gene conferring resistance to Cercospora sojina. Crop Sci 47: 1263-1 270.

Njiti VN, JE Johnson, TA Torto, LS Gray, and DA Lightfoot (2001) Inoculum rate influences selection for field resistance to soybean sudden death syndrome in the greenhouse. Crop Sci 41: 1726-1731.

Pace PF, DB Weaver, and LD Ploper (1993) Additional genes for resistance to frogeye leaf spot race 5 in soybean. Crop Sci. 33: $1144-$ 1145.

Phillips DV and HR Boerma (1981) Cercospora soiina race 5: A threat to soybean in the southeastern United States. Phytopathology 71: 334-336.

Phillips DV and HR Boerma (1982) Two genes for resistance to race 5 of Cercospora sojina in soybeans. Phytopathology 72: 764-766.

Schmutz J, SB Cannon, J Schlueter, et al. (2010) Genome sequence of the palaeopolyploid soybean. Nature 463: 178-183.

Shultz JL, S Kazi, JA Afzal, R Bashir, and DA Lightfoot (2007) The development of $B A C$-end sequence-based microsatellite markers and placement in the physical and genetic maps of soybean. Theor Appl Genet 114: 1081-1090. 
Smith TJ and HM Camper (1973) Registration of Essex soybeans. Crop Sci 13: 495.

Wrather JA, TR Anderson, DM Arsyad, J Gai, DL Ploper, A Portapuglia, HH Ram, and JT Yorinori (1996) Soybean disease loss estimates for the top ten producing countries during 1994. Plant Dis 79: 107-1 10.

Wrather JA, SR Koenning, and TR Anderson (2003) Effect of diseases on soybean yields in the United States and Ontario (1999 to 2002). Plant Health Progr (online Doi 10.1049).

Yesudas CR, H Sharma, and DA Lightfoot (2010) Identification of QTL in soybean underlying resistance to herbivory by Japanese beetles (Popillia japonica, Newman). Theor Appl Genet 121: 353-362

Yorinori JT (1992) Management of foliar fungal diseases in Brazil. p. 185-193. In L.G. Copping et al (ed.) Pest management in soybean. Elsevier Applied Science, London.

Yuan J, VN Niiti, K Meksem, MJ Iqbal, K Triwitayakorn, MA Kassem, GT Davis, ME Schmidt, and DA Lightfoot (2002) Quantitative trait loci in two soybean recombinant inbred line populations segregating for yield and disease resistance. Crop Sci 42: 271-277. 